\title{
Recording Meta-Soundscapes: \\ Synchronized multi-frame audio field-recording at a large spatial scale
}

\author{
David Dunn, David Kant, Matthew Mitchell, Ryan Page, Greg Sullo, \\ Mustafa Walker, Brian Ford, Jon Myers, and Madison Heying
}

University of California, Santa Cruz, Art and Science Research Group, Digital Art and New Media Program

Preprint, January 9, 2020

\begin{abstract}
As an investigation into alternatives to the predominant assumptions of conventional audio recording, the described research has sought to explore how field-recording of very large outdoor acoustic environments might be achieved. This was accomplished through real-time multi-channel recording from multiple locations using several surround microphone arrays, synchronized with wireless broadcast of conventional timecode. Such locations were as large as a half-mile in diameter. The resultant recordings of extended sound-fields represent complex auditory networks that are otherwise impossible for normal human perception to experience. The applicable technical details are outlined and several examples of their deployment put forth. Contrasting considerations for the application of these recording techniques to predominantly non-human versus anthropocentric sound environments are discussed. While this paper largely constitutes a report on preliminary investigations, it necessitates a broader discussion beyond its technical methods. A variety of aesthetic, perceptual, and scientific implications (including both explored and potential analytical methods) are presented as are strategies for multi-channel playback of the resultant recordings in installation venues or as binaurally encoded audio for headphone monitoring.
\end{abstract}

"Consult the genius of the place in all."

Alexander Pope

"Everything is new and doomed."

Tommy Orange 


\section{Introduction by the Principal Investigator}

Over the past half-century, through a wide variety of projects, I have been investigating the relationship of sound and music to the question of mind in nature. The research described by this paper has been a direct outgrowth and logical extension from those investigations. The resulting choreography of spatial microphone configurations and purposeful specification of personnel and resources might appear conspicuously similar to other compositions and projects that I have previously conceived, so why is this project so singularly regarded as a research project, per se? Perhaps the distinction is purely an academic conceit and one possible way of defining the role of the various participants, in light of the history of previous activity that informed this project, would be to equate them to performers in a more traditional musical context. However, this would be an incomplete characterization. While the role of the performer in much experimental music practice has become increasingly emancipated, performers are seldom asked to engage in serious discourse as to the meaning, substance, recollection of personal observations, and design of technical strategies that inform the projects in which they are participants. I am deeply grateful to my research associates for their diverse creative contributions and technical insights without which this project would not have been possible.

Of the various types of investigations that span my last five decades, these can be summarized as follows:

1) Site-specific performance events and compositions

2) Interspecies Communication

3) Soundscape and species-specific audio field recording

4) Data sonification

5) New audio transducer and circuit designs

6) Multi-channel surround and immersive audio displays

7) Bioacoustics research and interventions

Many of these areas of activity (4-7) had a well-established lineage that preceded my participation but others (1-3) largely started out at the experimental fringes of both the art and academic worlds. Some of the associated intellectual questions and creative outcomes that arose failed to garner either economic support or scholarly interest for decades. In fact, they were often met with direct ridicule even by other composers and artists associated with the "avant-garde." This was the case for both myself and for many other early practitioners. For example, I had the experience of being accused by the composer Cornelius Cardew - after listening to him praise Chairman Mao's Chinese Revolution - that what I and the Canadian Acoustic Ecology group were doing was simply an elitist attempt to prevent the common man from walking on the grass in public parks. While the recounting of that event certainly gives rise to an 
ironic chuckle, it may cause the reader to wonder-given all that has transpired regarding the direness of our current environmental circumstances — what was so controversial about these activities?

Many of these areas of creative and scholarly focus have subsequently morphed, in recent years, into mainstream research topics or areas of immense creative activity operating under the banner of Sound Art. Their current enthusiastic embrace by the academic field of Sound Studies is probably the surest evidence of their now acceptable - perhaps even fashionable — standing and validation that many of the questions, framed so long ago, still remain cogent. Since so many other artists and scholars have come to explore these ideas - with the ambition to further unpack implications at an ever-expanding rate-I no longer feel compelled to participate in the more readily familiar ways that I once did. Much of the creative sound art activities and their related Sound Studies research now constitute well-trodden and well-established paths. The subsequent deepening has been gratifying to observe.

However, I have also grown a little more circumspect about some of the underlying assumptions that now seem to predominate - and that I non-critically embraced — about the contribution that many of these activities (not to mention commensurate philosophical treatises) might make in advancing our understanding of the role of auditory perception in changing our collective relationship to our environment, sensitizing us to it, or in directly helping to solve environmental and social problems. Perhaps Mr. Cardew had the merest sprouting of a valid point. In fact, when engaged in non-critically, these activities can sometimes seem more symptomatic of the underlying problems that they purport to address than as practical aid. For instance, while an original intent of soundscape field-recording to be a strategy for increasing environmental awareness remains valid, it can, too easily, appear to be an art of high privilege enacted by those individuals who can afford the best equipment and plane fares to exotic places while shouldering the commensurate oversized carbon footprint without guilt. This is not an argument intended to negate such activities. To the contrary, my intention is to remind myself, and others, that these activities can be grounded in a clarity of purpose that transcends mere art-world careerism, especially if making claims to positive social impact. Hopefully, the reader may resist the temptation to lodge this same criticism at the project described by this paper. The assembly of such an aggregation of high-end audio gear may certainly appear intimidating to anyone, especially students, just starting to explore audio field-recording outside of a commercial context. While such access to extreme research resources may be one important contribution that still validates large institutions, it is also true that the current cost for such equipment is now a fraction of what it was even a decade ago. Notwithstanding the potential for appropriate criticism, this project does exemplify my current interest in both extending and challenging prior work and concepts. It now seems useful to explore a slightly different set of questions that seem more in-depth, with regard to issues of auditory and aesthetic perception, through heuristic 
methods - especially regarding our lack of understanding of temporal versus spatial perception — while also optimizing our use of the newest generation of audio tools and technical resources designed by artists and musicians that might facilitate and advance scientific research.

Amongst my prior investigations from the past half-century, one in particular seems especially relevant and might be described as an auditory epiphany. Here I consider it useful to repeat my previous description of that experience:

Several years ago, I spent time living on a houseboat deep into the Atchafalaya Basin of Louisiana. Nights were spent making forays into the swamp to record continuous night sounds without interruption. One of the most striking features of this sound world was the abrupt transition between distinct collectives of sound makers. One group would hold center stage for hours and then suddenly fade to silence. Within minutes a whole new cast of sonic actors replaced them. The dynamic quality of these dense soundscapes, with their fantastic spatial motion, impressed upon me a sense that - beyond the communicative agenda of individual living sound generators - there was some underlying emergent logic at work to drive them into a global patterning. It was as if there were multiple chains of communication linking together a fractally-delineated field of interlaced non-linear sources. These communicative chains not only extended outward in all directions but also up and down levels within a potentially infinite array of organizational hierarchies. No scientific study of such global interactions and feedback between diverse sound makers within an ecological network has ever been attempted.

In trying to attain a deeper understanding of such a complex dynamic, an obvious question arose: how might we even record sonic events at such a large spatial scale? When this question was first framed, the possibility of doing something akin to the research described in the current paper was inconceivable. Appropriate multi-channel equipment and surround microphone arrays were prohibitively expensive or simply did not exist. Furthermore, there required the rethinking, over subsequent years, of many of the well-established assumptions and inherent limitations of field-recording practices. Put simply, there are many kinds of sonic phenomena that cannot be adequately represented through traditional audio recording conventions. An excellent example is the collective sound of cicadas as an emergent global behavior. What most observers experience is a complex dynamical interaction between multiple individual soundmakers distributed over a large spatial distance. The phenomenon almost stands alone in nature for its acoustic depth and complexity. Stereo recordings fail miserably to capture the effect and single-point surround arrays (ambisonic) do only slightly better depending upon the accuracy and complexity of the playback circumstances.

The recordings that have resulted from this research project not only seem to subvert conventional norms of audio recording but also many of our conditioned perceptual expectations. This confrontation with the illusory nature of perception may be the project's most significant outcome and 
that, somehow, seems tied to the mystery of how and why we experience the phenomenon of time the way we do. While the classical view of Newtonian physics posits that time is fundamentally universal and absolute, alternate views of time have been debated by an important lineage of scientists and philosophers beginning with Leibniz and continuing on to Mach, Einstein, and many current thinkers. For example, the theory of general relativity asserts that time (as measured by a clock) is altered by the force of gravity. Two synchronized atomic clocks placed at different heights (floor versus tabletop) will eventually drift apart even with such a miniscule gravitational difference. It has become a consistent claim amongst physicists that our experience of "time's arrow" is illusory. An essential question that must be addressed by this research is, which of these contrasting views of time is the most appropriate for describing both the acoustic dynamics and perceptual complexities germane to this project? I have chosen to frame our observations in classical terms with regard to the experience of time, not because they are necessarily the best or most accurate formulations, but because they are the most familiar linguistic constructs congruent with our ordinary experience.

On its surface, this project may merely appear to be a technical investigation into novel audio recording techniques that have scientific implications but it can also be regarded as an artistic fulfillment of John Cage's polemic of allowing sounds to be themselves while also reframing that argument in the context of how those same sounds may represent mostly non-human communicative intent. An additional implication of Cage's aesthetic contributions is the assertion that it is possible to compose music and sound art by merely organizing an auditory perceptual circumstance independent of any intentional sound-making. This implication also points to events within visual art history embodied in the work of James Turrell and others: making visual art that primarily consists of the mere materiality of existent light, space, and perception. This project posits one possibility for an auditory equivalent using only the capture (and playback) of existent sound that challenges our perceptual expectations and habits.

\section{David Dunn}




\section{Multiple Frames of Reference and a Bird's Ear Audition}

Imagine what several people spread outdoors at a substantial distance from each other are hearing simultaneously, as if all of their ears were somehow superimposed on top of each other. In contrast, imagine a singular lifeform capable of hearing almost everything that is happening in a valley or canyon all at once from a unique perspective. While these two scenarios are very different ways of framing the problem of how sound behaves and articulates extended space, neither of these scenarios are germane to the everyday concerns and paradigms of conventional audio engineering. The underlying principles of "proper" sound recording are well-established even though there are divergent ways in which those principles are applied. However, the aforementioned thought experiments may have a useful bearing upon questions of how our perception of time and space unfolds and how that unfolding may contribute to the use of sound to study and understand a range of environmental phenomena.

Traditional audio recording presupposes a point of audition (POA) similar to the point of view (POV) of photography and cinema. The creation of stereo or surround recordings results from two or more microphones properly placed such that phase and amplitude are sufficiently conformed to an ideal individual or group listening position. Ultimately this is a necessary contrivance that seeks to encode spatial and temporal information correlated to the original sonic events. It is often desirable to adjust phase differences that result from the speed of sound over varying distances. For instance, in a typical concert recording, where room mics are placed further away from the sound source than the spot mics that are much closer, it can be necessary to delay the audio signal of the spots to make the phase alignment with the room mics more congruent. This can be desirable to reduce unwanted destructive interference. The consistent factor in almost all recording scenarios is that the final recording will be heard by listeners in some ideal POA (the so-called "sweet-spot"), whether that consists of a living room, movie theater, or concert hall.

When microphone positions exceed the possibility of phase normalization due to extreme distances, the anomalies that emerge would ordinarily be considered highly undesirable and incoherent when collapsed to the standard assumption of a singular POA. By and large, this project represents an overt challenge to such technical and perceptual norms. An essential assumption underlying this project has been that time alignment must be precise in order for the recordings, made via these relatively imprecisely placed microphone arrays, to maintain both aesthetic and analytical relevance. Even if the spatial placements of the microphone arrays are imprecise, information about their spatial relationship is encoded in the recording as long as the time reference is precisely synchronized (electromagnetic waves moving at light speed) and each microphone recorded to a separate channel. The concept of a single POA is entirely dispensed with in both the recording and playback circumstances. Collapsing the large outdoor space into 
an indoor playback environment, while recreating a general correlation to the original recording positions through multiple loudspeaker placements, creates a compression of space that allows for the perception of these multiple points of audition. Stated simply, conventional recording techniques can often compress temporal reality to conform spatial distance whereas, in this approach, we precisely synchronize temporal reality in order to compress large spatial distances. The experience invites a listener to move through the space to perceive local nested details while still hearing the total soundscape that surrounds them.

\section{Technical Strategies and Solutions}

While our research primarily focused upon developing and testing methods for recording large acoustic outdoor environments from multiple microphone positions, spaced apart at great distances, an additional research issue addressed the question of what do we apprehend by a comprehensive listening (informed by aesthetic considerations) to complex sonic data as opposed to only applying analytical methods? Our approach to investigating this question was to concentrate on how a large acoustic environment might be captured from multiple points in space with very high-resolution microphones, sampling rates, and sample-accurate synchronization. Therefore, in all of our research situations, the highest practical resolution was desirable. This consideration not only applied to the use of very highquality microphones for each array placement but also the use of high-quality, multi-channel digital audio recorders that could support sample-accurate timecode synchronization. All recordings were made with $96 \mathrm{kHz}$ sampling rates at $24 \mathrm{bit}$ resolution.

A survey of the various microphone arrays, microphone models, digital audio recorders, and timecode technology used during the research, in a variety of combinations and circumstances, is given in Table 1 and Table 2. A Zoom F8 recorder always served as the source timecode generator connected to the Azden transmitter. The broadcast of this master timecode was sent to individual receivers (tuned to a common frequency) attached to each of the other recorders, all set to slave mode in order to synchronize with the master machine. Each separate multi-channel recording was later aligned with all others by matching their unique start times to the master timecode and the timeline indicated by their meta-data files. 


\begin{tabular}{|l|l|}
\hline \multicolumn{2}{|l|}{ Microphones } \\
\hline Binaural (human head-type type mounting) x 2 & 4 DPA 4060 \\
\hline Jecklin Disk stereo x 2 & $\begin{array}{l}\text { 2 Sanken C100k } \\
\text { 2 Sennheiser MKH20 }\end{array}$ \\
\hline Ambisonic x 3 & $\begin{array}{l}\text { 3 Sennheiser Ambeo } \\
\text { Double MS (DMS) x 2 Sennheiser MKH 800 twin } \\
\text { 2 Sennheiser MKH30 }\end{array}$ \\
\hline 5.1 integrated array x 1 & DPA 5100 \\
\hline INA5 (modified) x 1 & $\begin{array}{l}\text { 2 Sennheiser MKH40 (L\&R) } \\
\text { Rode NTG3 (C) } \\
\text { Crown SASS Mk2 (rear L\&R) }\end{array}$ \\
\hline IRT Cross x 1 & 4 Blue Hummingbird \\
\hline 180-degree stereo x 1 & 2 Rode NT1A (modified capsules) \\
\hline
\end{tabular}

Table 1: Microphone array configurations and microphone models used.

\begin{tabular}{|l|}
\hline Digital Audio Recorders \\
\hline Zoom F4 x 6 \\
\hline Zoom F8 x 3 \\
\hline Tascam HD-P2 x 2 \\
\hline
\end{tabular}

\begin{tabular}{|l|}
\hline Timecode Broadcast \\
\hline Azden WR-PRO Wireless Receivers x 10 \\
\hline Azden WM-PRO Wireless Transmitter x 1 \\
\hline
\end{tabular}

Table 2: Digital audio recorders and timecode technologies used.

\section{Three Applied Examples}

While several recordings were made during the period of this research, a few were unsuccessful due to unfavorable environmental conditions or technical failures. Three others were especially successful and emblematic of the recording process in ways that best exemplify the project as a whole. These recordings not only represent the technical strategies being tested but also generated novel insights into the unique characteristics of each location: 


\section{1) Horseshoe Lake}

On April 14, 2018, a moderately-sized lake in the Santa Cruz Mountains, near La Honda, California, was recorded from six positions. The lake is roughly 0.5 miles in diameter. Although its shape vaguely reflects its name, it is fairly asymmetrical and nested within a mixed conifer and deciduous forest ecology. Most of the geological features extend out from the lake's flat terrain with a large hill extending upward from the inner dip of the horseshoe on one side of the lake.

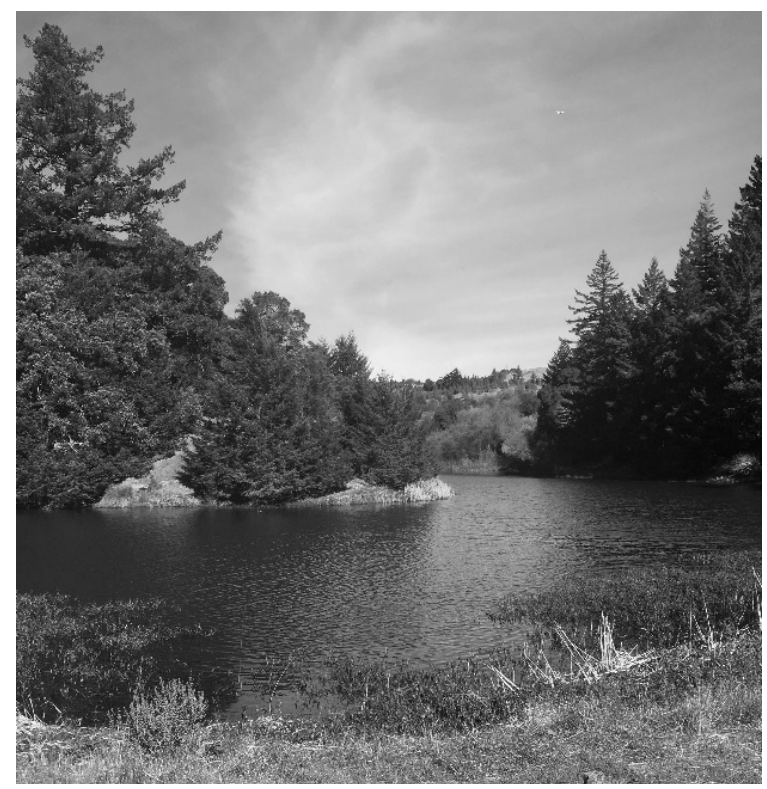

Photo 1: View of Horseshoe Lake from position A.

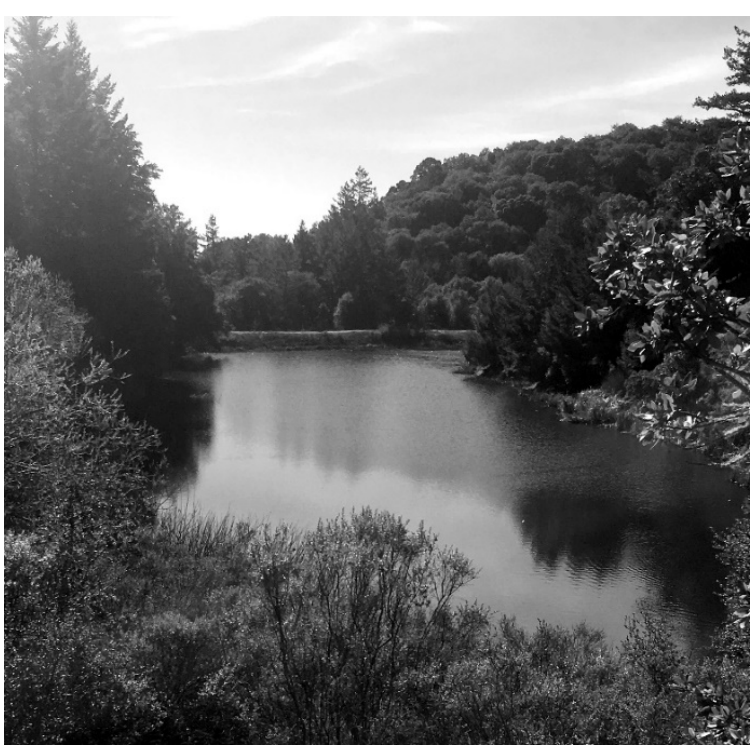

Photo 2: View of Horseshoe Lake from position F. 
The six positions of the microphone arrays and their microphone types are indicated by Figure 1. Two Zoom F8 recorders and four Zoom F4 recorders were used at the various microphone positions. The center 5.1 and binaural arrays were recorded using a F8 recorder that also served as the master for timecode broadcast. In addition to the center arrays, three ambisonic microphones, and two Double MS arrays were deployed. The recording began around $3 \mathrm{pm}$ and lasted 2 hours and 15 minutes. A wide variety of sounds were recorded during this timeframe including those of insects, various waterfowl, many other species of birds that were both stationary and in flight, amphibians, humans hiking the trail that surrounds the lake, and other anthropogenic sounds such as cars, motorcycles, aircraft, and an anonymous improvising violinist somewhere in the distant forest. Each microphone array picked up sounds that were shared by those positions close enough to a common sound source but also unique sounds not shared by other positions.

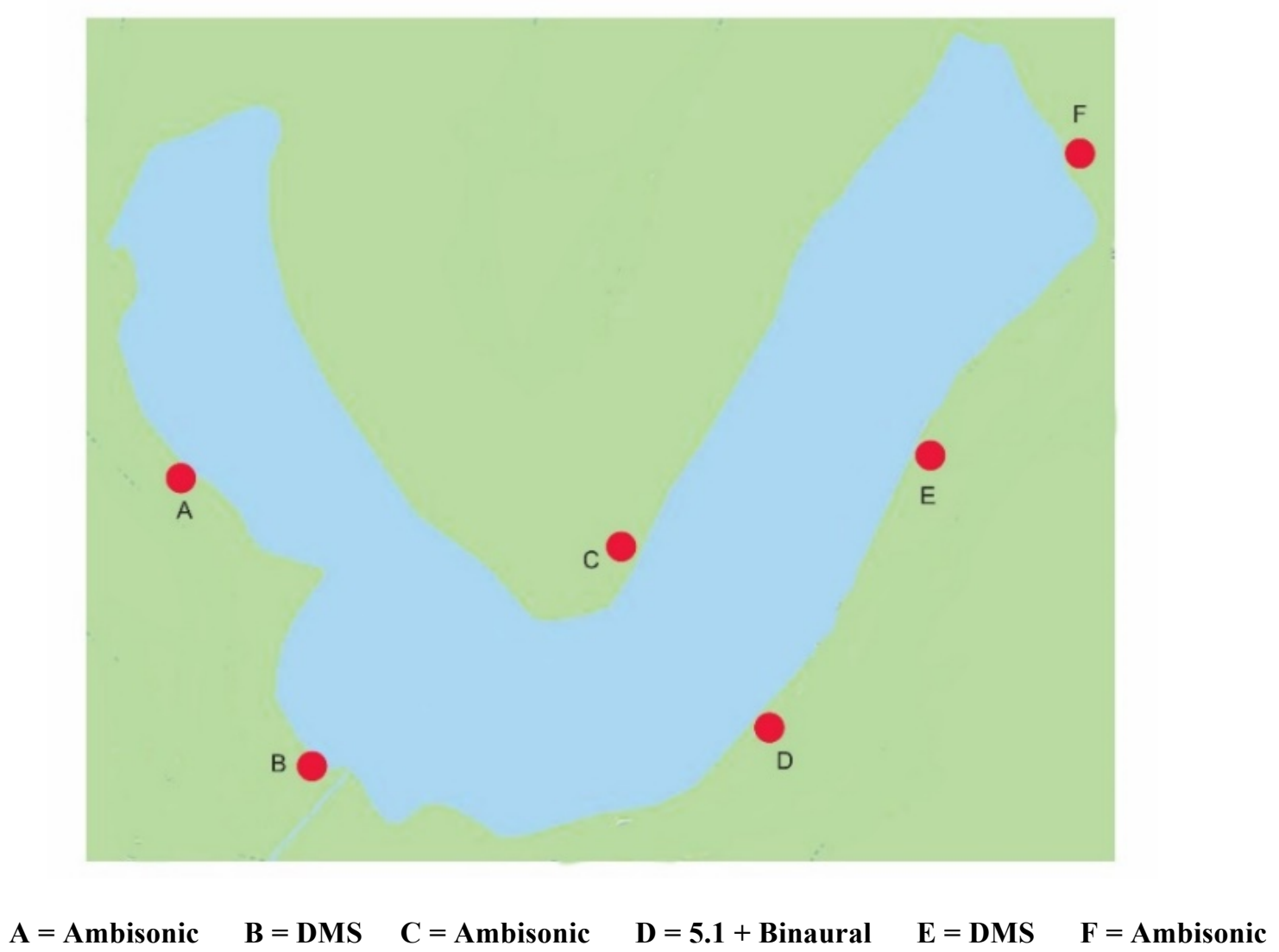

Figure 1: The six microphone arrays as positioned around Horseshoe Lake. 


\section{2) Alpine Pond}

On October 27, 2018, a pond environment, in the same general area as Horseshoe Lake, was recorded from nine stationary locations and two more in motion as indicated by Figure 2 . The stationary microphone arrays were placed as symmetrically as possible in order to completely surround the moderately-sized pond. The two arrays in motion started at the same location and were then slowly carried in opposite orbits around the entire circumference of the pond. The entire recording took one hour. A similar assortment of sound sources as those at Horseshoe Lake were recorded during this time period. However, the recording began approximately a half-hour before sunset such that the final result reflects the transition of many daytime sound-makers into those of the early evening. While this transition is gradual, it is also quite dramatic.

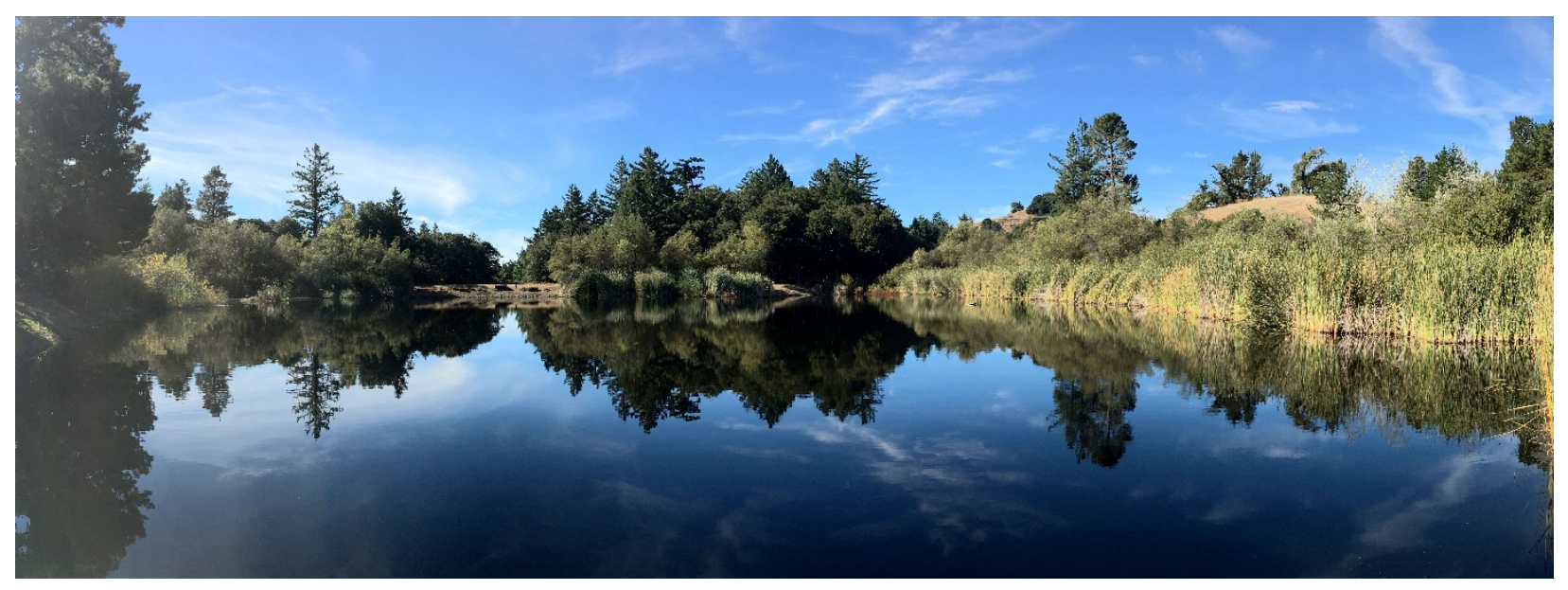

Photo 3: Wide view of Alpine Pond.

The mobile recordings used two ambisonic microphones and Zoom F4 recorders. The symmetrically placed stationary arrays consisted of two Jecklin Disk stereo setups with Tascam HD-P2 recorders, two Double MS arrays with Zoom F4 recorders, two binaural arrays with Zoom F4 recorders, two 5-channel arrays with Zoom F8 recorders, and a larger array of an IRT Cross and a 180-degree stereo pair, with a Zoom F8 recorder, placed as an asymmetric offset to the otherwise circular placements. The offset Zoom F8 recorder also served as the master for timecode broadcast. 


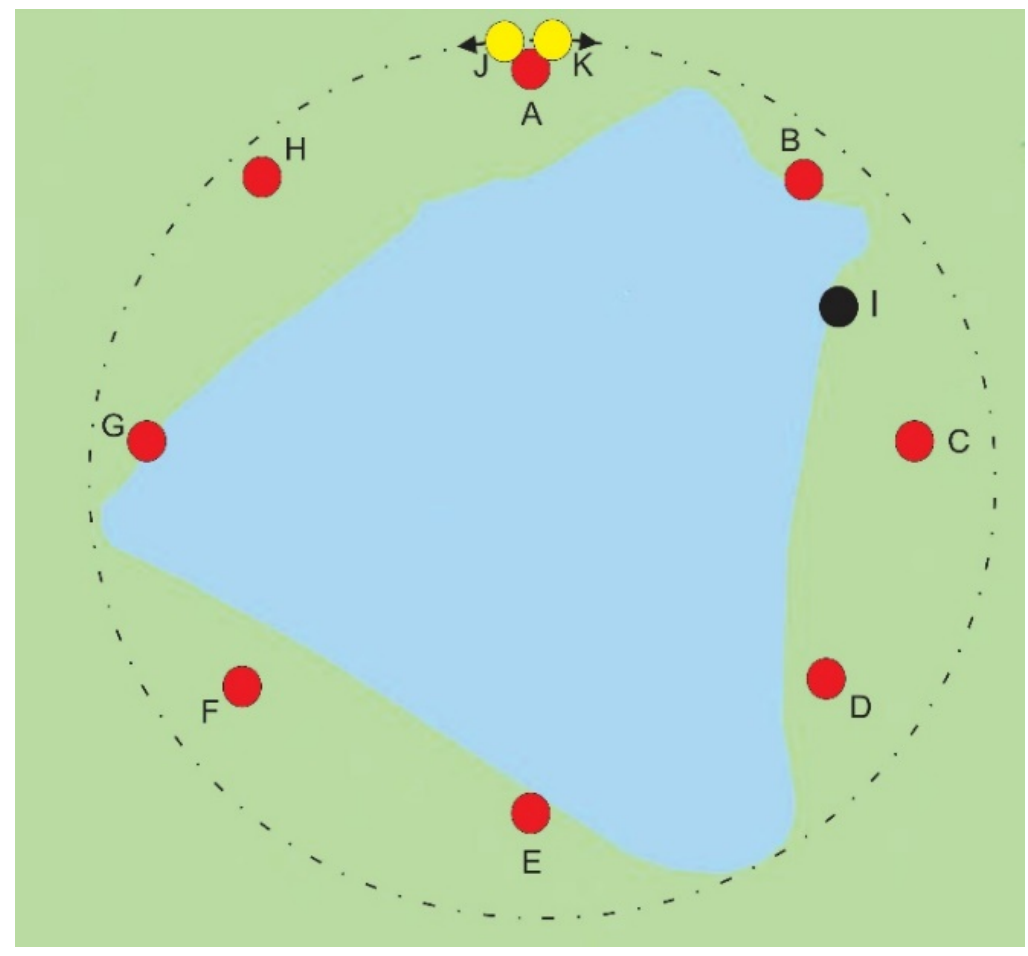

\section{A $=$ 5.1 Array $\quad B=$ Jecklin Disk $\quad$ C $=$ DMS $\quad$ D $=$ Binaural $\quad E=5.1$ Array $\quad F=$ Jecklin Disk \\ $G=$ DMS $\quad H=$ Binaural $\quad I=I R T$ Cross +180 Stereo $\quad J=$ Ambisonic $\quad K=$ Ambisonic}

Figure 2: The eleven microphone arrays as positioned around Alpine Pond.

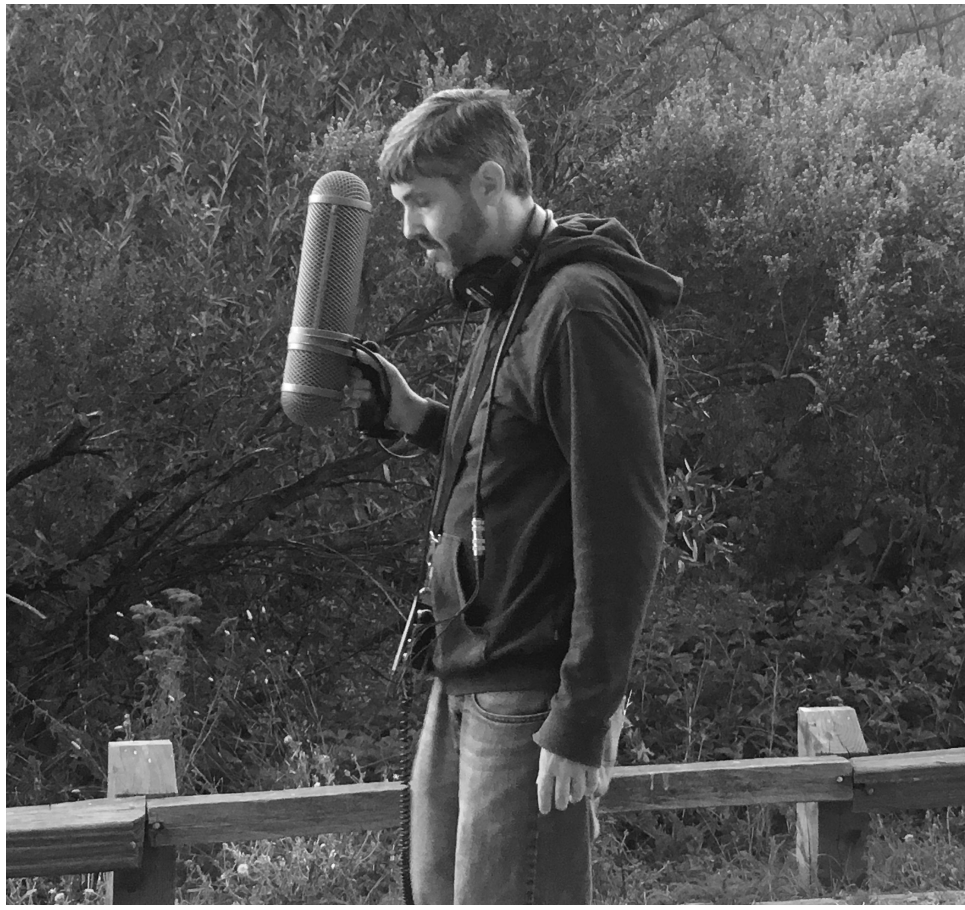

Photo 4: Matthew Mitchell carrying an ambisonic array while in motion at Alpine Pond. 


\section{3) UCSC Campus}

On November 6, 2018, an open transit space between campus buildings was recorded from five stationary locations. The area includes multiple large architectural features, a parking lot, and a small sloping hillside, all punctuated by numerous large trees and other foliage. While both car and pedestrian traffic are predominant, the space also hosts intermittent bicycle and skateboard activity. Virtually no non-anthropogenic sounds occurred during the 1-hour recording duration even though the UCSC campus is rich with wildlife that daily traverse through the campus at large.

A master Zoom F8 recorder and an 8-channel microphone array, consisting of an IRT Cross and an ambisonic microphone, were placed in the center of the space. Two Jecklin disk stereo arrays and two Double MS arrays, all with Zoom F4 recorders and timecode receivers, were placed at similar distances surrounding the central array. This configuration is shown in Figure 3.

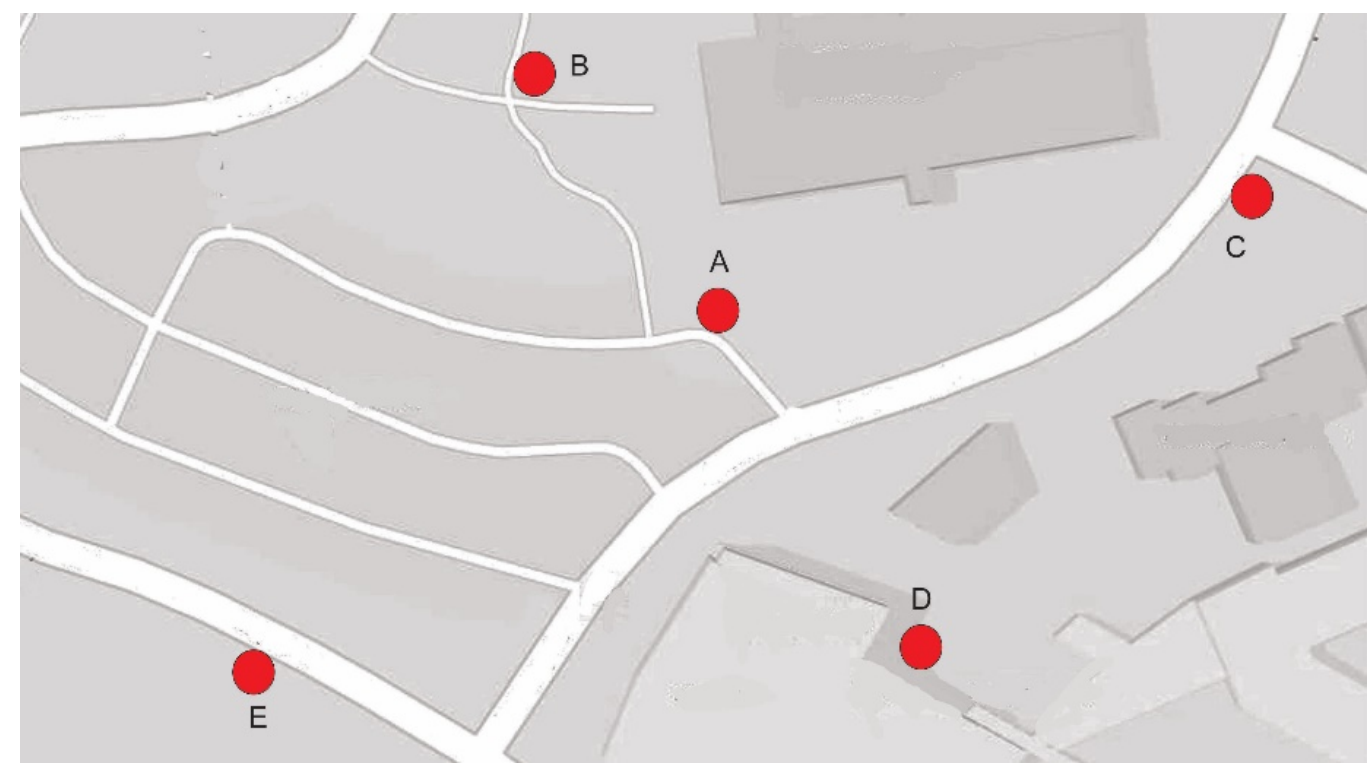

\section{A $=$ IRT Cross + Ambisonic $\quad B=$ Jecklin Disk $\quad C=$ DMS $\quad$ D = Jecklin Disk $\quad E=$ DMS}

Figure 3: The five microphone arrays as positioned on the UCSC campus (between the Music Center and the Digital Arts Research Center). 


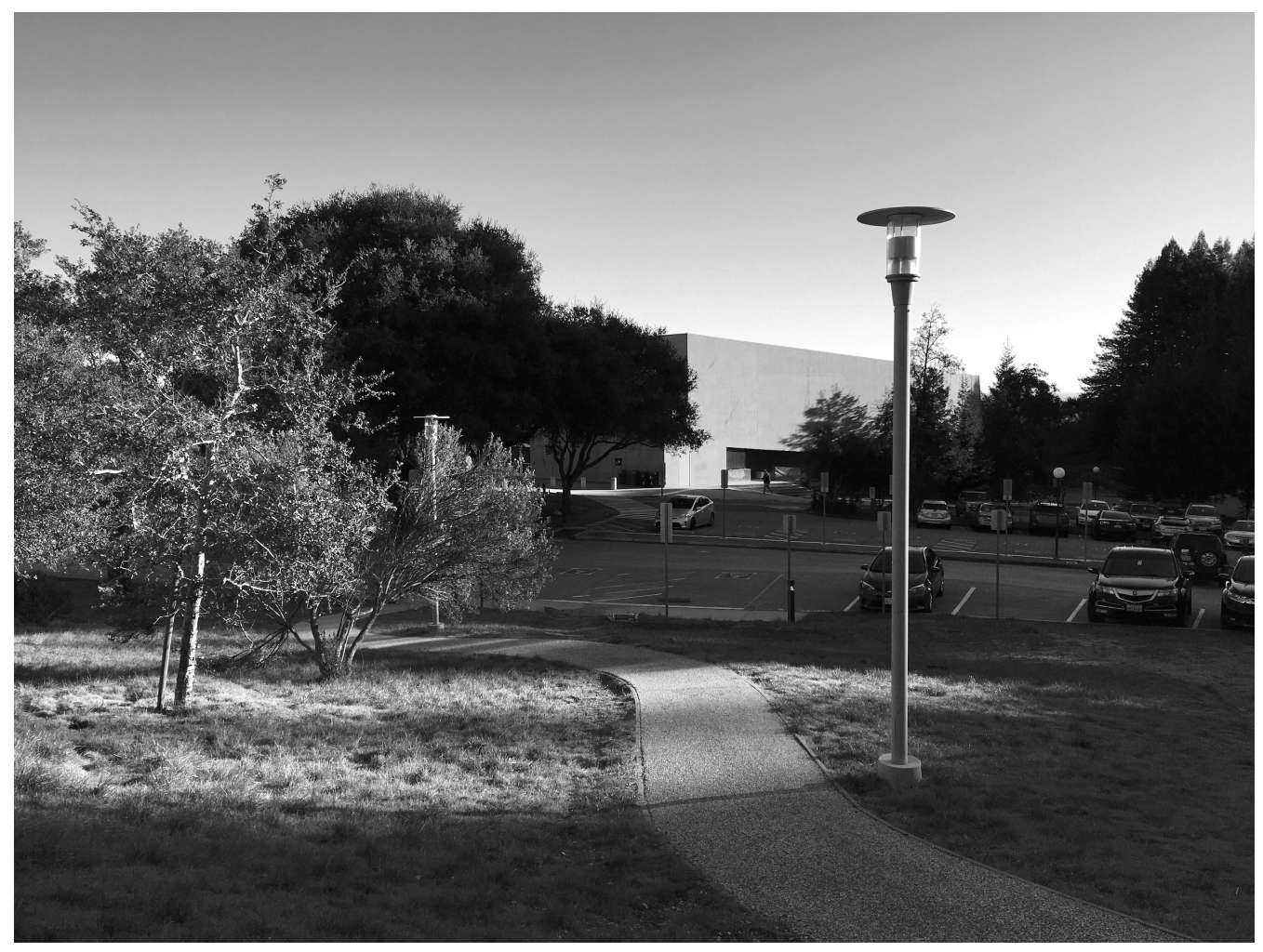

Photo 5: View of UCSC Campus from position B.

\section{A Hearing of Many Hearings}

What is the final effect of the recordings? Upon much listening and discussion of these recordings, our research group has come to hear in these recordings a surprising emergent presence that is unusually symphonic when compared with the more familiar techniques for soundscape recording or bioacoustics research. A central research assumption, banal by the standards of conventional stereo soundscape recording, was that none of the sounds captured by the recording process were to be edited. A conspicuous multi-dimensional and immersive depth is evident that takes on a kind of "holographic" presence beyond familiar surround sound strategies. How might we account for this non-ordinary experience?

In a typical concert recording, the final product results from the complex interaction of sounds within an enclosed acoustic space. Multiple reflections converge to create both constructive and destructive wave-fronts that determine what is distinctly heard at any particular POA in the room. Both constructive and destructive interference can be of equal importance to shape what we will refer to as spatial resonance (the combined reflections that give rise to room acoustics at a single frame of reference). 
In large outdoor acoustic spaces that are devoid of human structures (generally referred to as "freefield spaces"), there is minimal constructive or destructive interference due to reflective surfaces. The most notable exception being landscape features that result in substantial echoes (significant instances of constructive interference due to very long wavelengths will be discussed in a later section). In this circumstance, sound events travel outward as an expanding sphere with few impediments to create dramatic delays when referenced from multiple frames of reference (multiple microphone arrays placed at large distances that delimit most acoustic interference). Here we will use the term temporal resonance to characterize the circumstance of multiple recordings of a shared sound source substantially splayed in time and their perceptual attributes caused by the speed of sound over distance. As a result, it is often difficult to distinguish between the resultant time and distance echoes-caused by the juxtaposition of multiple frames of reference - from echoes resulting from landscape features. Since there is so little destructive or constructive interference, a different kind of "resonance" takes shape that is multidimensional in its spatiotemporal complexity. It challenges our preconditioned experiences about hearing and listening. The novelty is both exciting and confusing.

Figure 4 illustrates some of the differences between traditional recording techniques and what we will refer to as Free-field Multiple Frame recording as demonstrated by this research project. Normal stereo recording techniques attempt to capture a spatial sound field in a mostly horizontal plane. Depth and height information are largely diminished. The stereo array is generally limited to the separation width between two microphone capsules oriented to a desired sound source. Ambisonic recording extends traditional stereo techniques to a four-capsule array with the capsules usually positioned in close proximity as a tetrahedral configuration. The assumption is that the four signals produced by this array can represent a sphere of sound converging to a single point in space. The resultant multi-channel audio signals can then be combined in a variety of ways so as to produce flexible stereo representations and/or a large choice of surround playback options. To summarize:

Stereo $=$ Selective source of sound propagating towards a single point in space (POA)

Ambisonic $=$ Sphere of sound converging to a single point in space (POA)

Free-field Multiple Frame = Many synchronized positions (POA) spread over a large spatial scale 

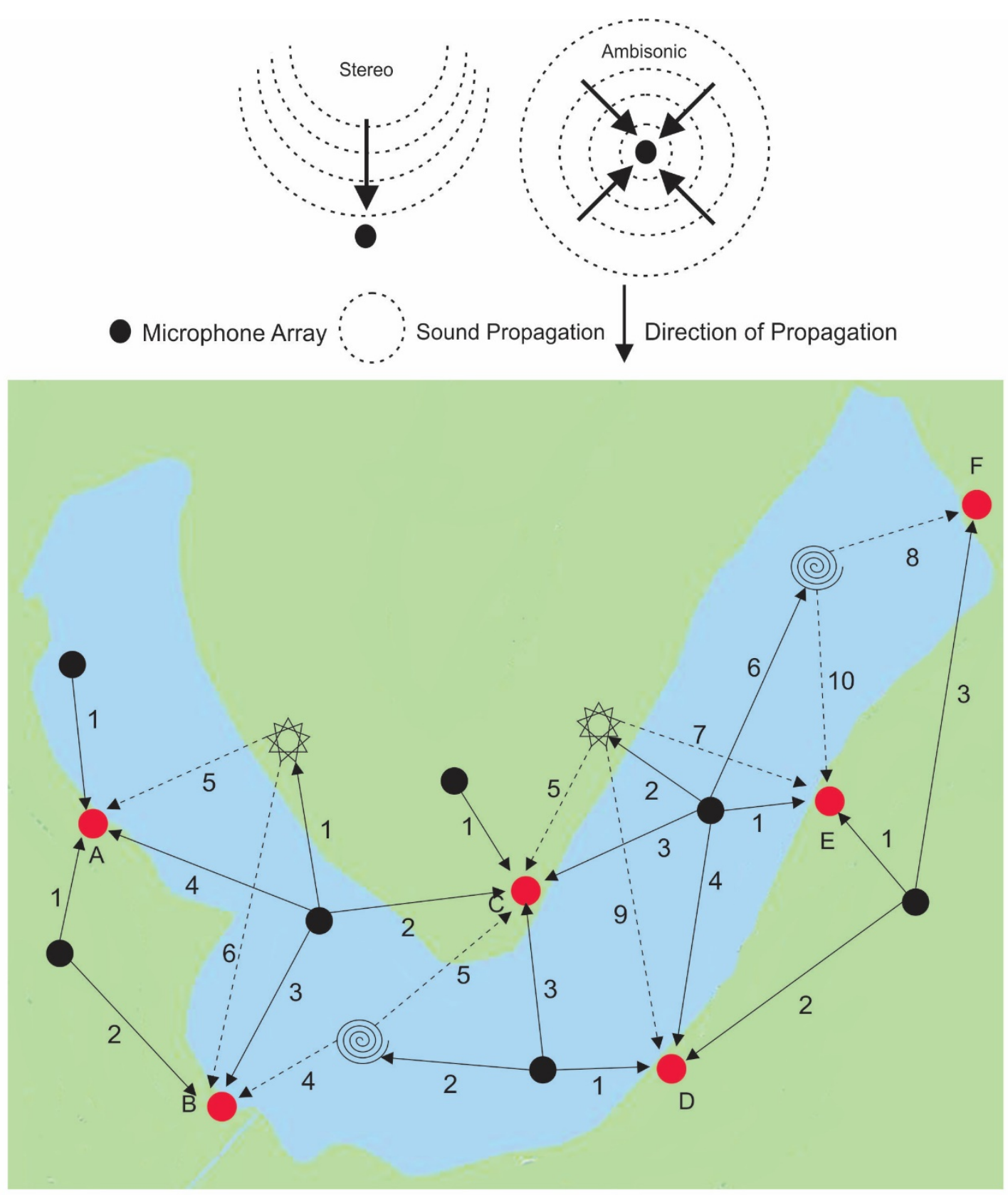

\section{Free-field Multiple Frame}

One hypothetical temporal configuration showing multiple synchronized microphone arrays but independently occurring sound sources. Possible sound arrival times and their directions of propagation are indicated as are some potential reflective surfaces.

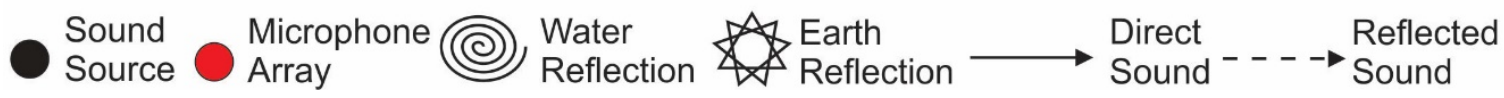

Figure 4: Comparison of three audio recording techniques and their effective representation of spatial scale. 


\section{Playback Considerations}

The successful recording of these large outdoor spatial locations into multi-channel audio files is just the initial technical problem to be considered. How might such a complex auditory representation of extended space be made apprehensible when rendered as a listening experience through auditory playback?

There is a history of experimentation with spatial audio playback that challenges conventional surround sound solutions based upon the underlying assumption of a singular POA and its commercial standardization. These alternative ideas have mostly been advanced as either art performance events or gallery/museum installations that use a unique distribution of sound sources. These strategies assume that listeners will explore the acoustic space as active participants rather than as observers confined to a static POA.

An excellent example of this approach is the classic sound installation, Rainforest, by composer David Tudor. A variety of everyday physical objects and/or junk constructs are distributed throughout a gallery or performance space. They are each activated by acoustic drivers that function as non-linear

loudspeaker systems through which electroacoustic audio signals are amplified. The "audience" is encouraged to move around the space in order to visit these sounding constructions up close, or at a distance, as the whole sound environment takes on the intended effect of its title. The experience is immersive and complex.

While we have been exploring multiple strategies for such complex distributions of playback sources, here we describe one example that has been particularly successful at compressing the sense of original spatial scale into an interior room. In Figure 5, we see the placement of multiple loudspeakers and other playback devices. This assumes a collapse of the original 26 audio channels of the Horseshoe Lake recording to 20 playback channels. The three ambisonic microphones, two double MS arrays, and multi-channel 5.1 (plus binaural) array, were each collapsed to binaural output channels and force panned to relevant loudspeaker pairs that roughly reflect their original recording positions. In addition, all six recording positions were played back as binaural outputs to six pairs of foam-core boards, sonically activated by attached audio exciters. These devices allow listeners of various heights to stand between the parallel boards and binaurally experience what a person standing at that location might have heard, while still hearing all of the multiple locations as surrounding audio. The amplitude of the foam-core output transducers is much lower than that of the 8-channel surround system but become very effective as a kind of sonic focusing lens when someone stands between them. 


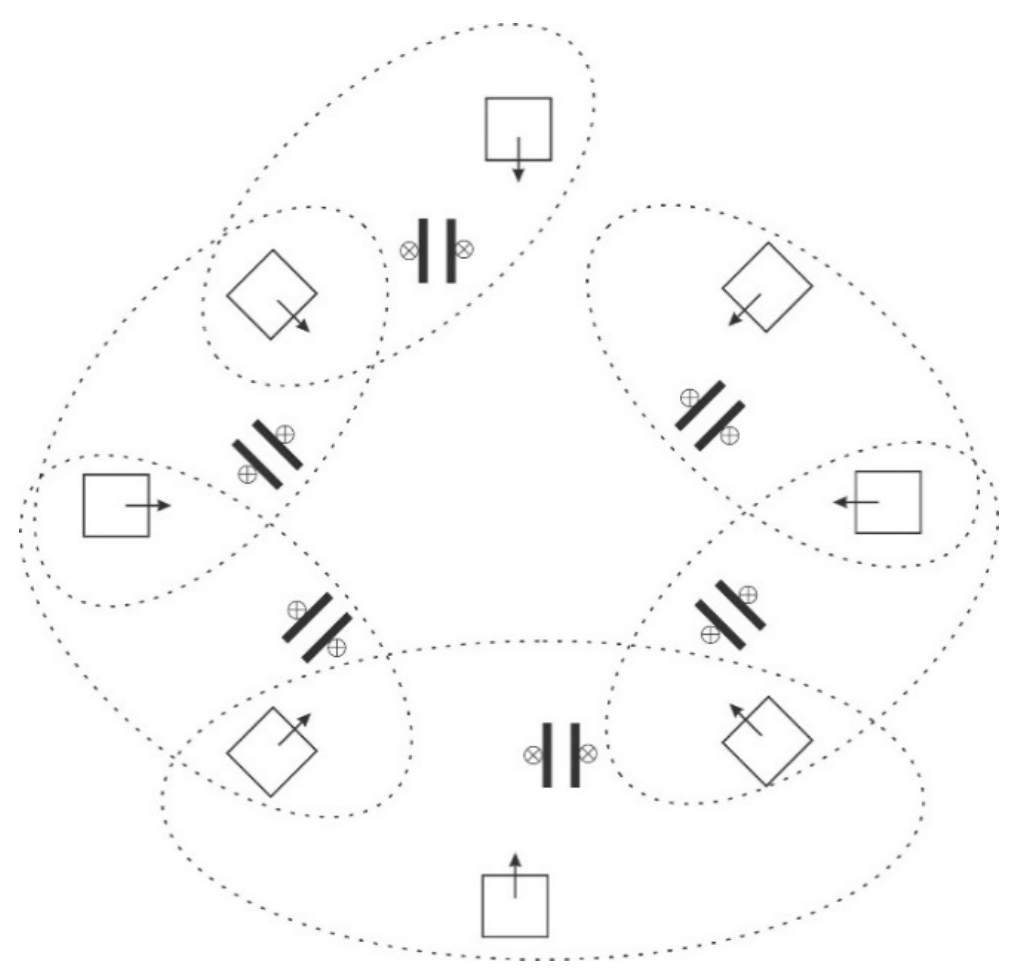

Figure 5: Experimental surround setup for the playback of the Horseshoe Lake recordings. Conventional loudspeakers are shown as rectangles while the exciters mounted on foam-core boards are shown as parallel lines and small circles. Audio channel assignments of binaural sub-mixes are shown by large ovals with broken lines.

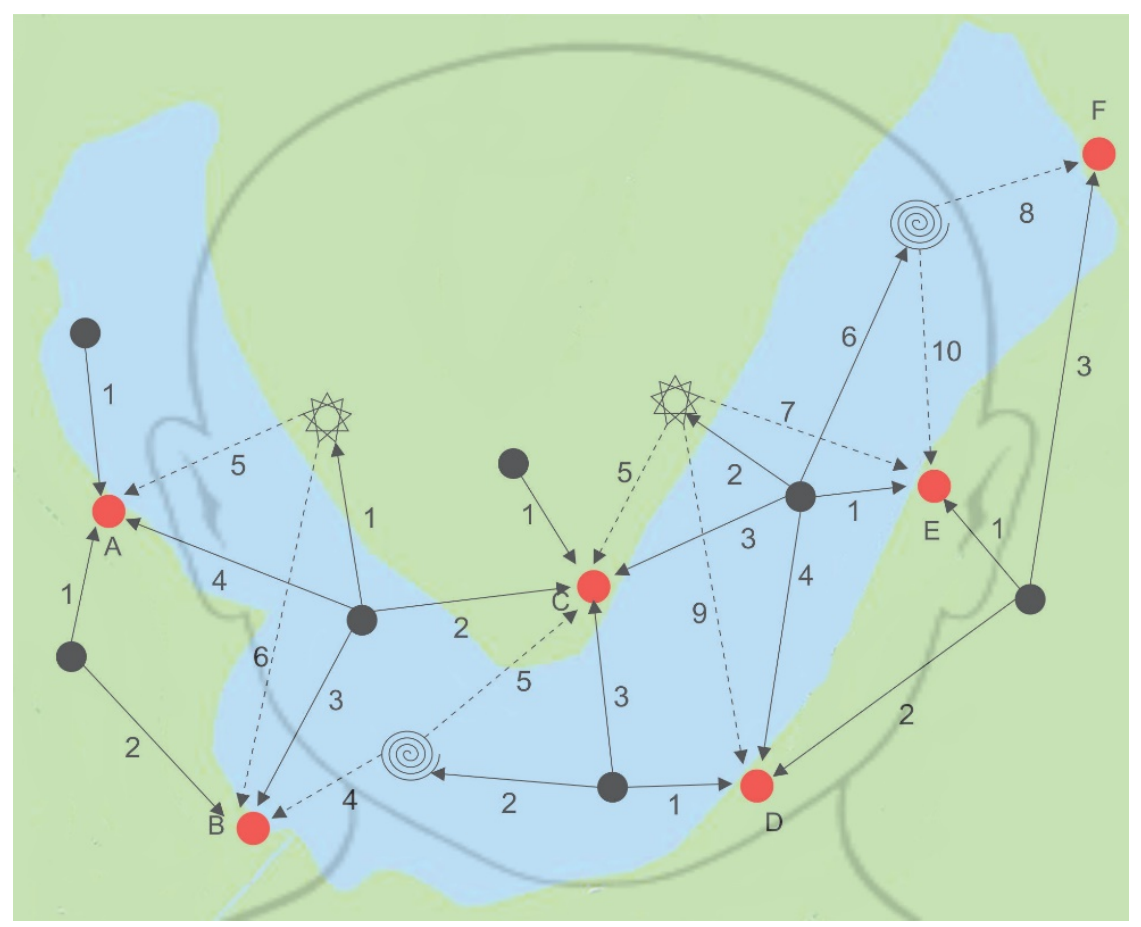

Figure 6: Impressionistic representation of the binaural listening strategy. 
A very different playback solution was also explored that is, in many ways, the inverse of the installation version described above. Rather than distributing the multi-frame audio information throughout an open exhibition space, this solution collapses the multiple audio channels into a binaural mix for headphone listening. Figure 6 shows a suggestive impression of this binaural listening strategy. The six microphone arrays that were placed across the lake's half-mile diameter distance were placed into a contrived binaural auditory field. The recordings from the original central positions (C and D) were retained as the primary binaural reference placements. The other arrays (A, B, E, and F) were each collapsed to monophonic mixes with each "hard panned" separately to their corresponding spatial positions within the headphone playback stage (left to right). The resultant listening experience is more akin to that of a singular listener with access to an impossibly wide auditory field made apprehensible through a final 2-channel audio mix.

\section{Wild versus Urban Soundscapes: Anthropogenic Sound within the Non-human World}

There is certain value to philosophical arguments that point to a false dichotomy posed by the use of the term "nature." Its historical usage has largely assumed a problematic distinction that removes humans from the underlying network of processes that define our existential reality. However, there remains a categorical and experiential difference between the human and non-human worlds that is made apprehensible through this research. Perhaps the word wild is a better substitute for expressing this categorical difference. The poet Gary Snyder has defined this concept in an essential way: "Wildness is process. It's just a name for the process of the impermanence and flow of change of phenomena as constantly going on without human intervention."

This categorical difference is readily apparent between the two kinds of recorded environments explored by this project. One of the most conspicuous features of the urban recordings is that they tend to exhibit a lack of underlying coordination between the sound sources. Humans are going about their individual business with only an ancillary relationship to others. Any auditory communicative intent is mostly limited to speech occurring at close range or into an electronic device such as a cell phone. Most of the sound-making is not communicative at a distance except, perhaps, car horns or the less common event of people shouting to each other or making music. There is no overt sense of a conscious participation in a larger fabric of sound or with anything that is other than the human wrought environment. The urban recordings also display a degradation of the complexity of audible soundscapes due to masking effects produced by constant traffic or buildings with mechanical systems such as HVAC, lighting, and the electrical grid. 
By contrast, the predominantly wild sound recordings exhibit a kind of integrated richness. The myriad living sound-makers reveal a need for mutual awareness. You hear the call and response of individual organisms communicating at a substantial distance and a constant responsiveness to changing factors in the environment. It is clear that these recordings evidence living communicative networks. The presence of human sound-makers become either part of those networks or an external sonic envelope that perturbs the local biome along with the ancillary sounds of other non-living sound sources. Borrowing from Gregory Bateson's concept of a cybernetic definition of mind, we might characterize these recordings as descriptions of an emergent mental system. We might also metaphorically imagine that they tell us something about how that mentality hears itself. These sounds are the evidence of intentional communicative exchange within a living fabric of mind.

There are also purely physical characteristics that distinguish these two different acoustic worlds. In the wild environments the aural communicative network exhibits a preponderance of medium to short wavelengths that are evident as out of phase delays articulating distance between microphone arrays. Very long wavelengths are often constructive between arrays while short wavelengths are largely neither constructive nor destructive because of much less wave-front interference. In-phase sounds that are constructive between multiple mic positions are mostly produced by human sources with long wavelengths and high amplitudes. Very long wavelengths can become additive to multiple arrays because they are more in phase over large distances. Sonic events that are higher in elevation can also be more equidistant to the various microphone arrays while events in the same plane seldom are. The reader can contemplate the difference between a hawk circling above the entire acoustic space (fairly equidistant to the mics below) versus a duck circling on the surface of a pond (less equidistant to the mics in its same horizontal plane). Short wavelengths create dramatic delays because they are out of phase relative to spatial distance. This creates a spectrum of phase alignments that range from additive to splayed time delays but much less potential destructive interference when compared to reflections from external architectural surfaces or enclosed spaces (indoor rooms, concert halls, etc.).

In human urban environments, long wavelengths can be constructive but short wavelengths can commonly be either constructive or destructive through architectural reflections. There are also fewer short wavelength sounds of moderate to high amplitude. When they occur, they are mostly audible at close proximity (speech or footsteps, for example). Exceptions to this would be those sounds that are intentionally communicative at a distance (shouting, car horns, music, amplified speech). What predominates are the long wavelength, high amplitude sounds of traffic, HVAC, and other machines. This, of course, is all relative to the kind of frequency/amplitude spectrum that is manifested under constantly varying conditions. 


\section{Perceptual Implications and Conundrums}

The detailed, three-dimensional aural picture created by each individual array position-when played simultaneously_presents several distinct phenomena that result in a number of disorienting deviations from how one "normally" perceives and makes sense of aural information. For instance, the sense of relative size and proximity become unfixed. Unrelated to changes in overall amplitude, the perceived size of sounding agents can abruptly shift in relation to their movement in the recorded environment. The playback space becomes a virtual environment that not only truncates distance but results in perceived non-linear motion: movement of the same sounds to and from multiple locations and an intermittent switching between the perception of sounds within the virtual recorded environment and their physical placement within the installation. Overall, the perceptual and cognitive tools that are typically relied upon to make determinations about acousmatic sounds become unreliable. Depending on the position of the listener-determining the perceived volume and relative position of certain soundsinformation from each of the distinct fields of listening takes precedence. While masking is a common phenomenon in recorded audio, here the masking creates distortions that are unique and difficult to describe. This phenomenon requires a new vocabulary and taxonomy as well as methodology for relating the quantifiable aspects of the sounds to their empirical perception. The issue may be an auditory equivalent to what is known as the size invariance problem in cognitive psychology. How does a child learn to understand that their mother's face remains the same even though its scale changes? It appears large when close but small at a distance. We simply do not know how the child's brain learns to resolve the problem.

Lest we forget, audio recordings are a form of representation and description of sonic events in reality. The sensorial satisfaction that is achieved can mask the fact that the process is a technological mediation with a communicative intent. It can be too easy to confuse the map for the territory. As the classic TV commercial asked: "Is it live or is it Memorex?" However, the recording process actually collapses our auditory reality to a focused form of attention that is somewhat equivalent to the flattening of visual experience that takes place with film or video technology. Every soundscape recording is a contrivance that describes some aspect of the world and not a recreation of it. Choices are being made that draw attention to aspects of reality that are otherwise not available to listeners or impossible to perceive without technological mediation. What differentiates this form of representation from the everyday linguistic domain that we occupy?

Jean Piaget was the first to extensively study children's conceptions of time and space. While he found that "time and space form an inseparable whole" in the child's mind, he also claimed that there was an apparent imbalance between how they could express the relationship between time and space in the 
linguistic domain. Cognitive science has since reinforced this insight through multiple experiments that show a cross-dimensional asymmetry between our mental representations of these two dimensions of experience. This is true for both children and adults. Temporal representations are more dependent on space than the other way around. Not only do people mostly use spatial words to talk about time, they also seem to use such spatial constructs to think about it. Is this cross-dimensional asymmetry only confined to the linguistic domain or does it carry over to how we construct and perceive recorded audio representations of reality?

Audio representations do seem different in how they represent and influence our cross-dimensional perception of space-time. Audio reproduction may often emphasize time over space, especially when we collapse, truncate, or almost make irrelevant, the spatial dimension to the listener's perceptual awareness. This most often occurs with the need to conform the listening experience to the convention of a singular POA. While conventional surround sound strategies strive to enhance spatial information, they mostly increase information about the position of sound sources but much less about their distance. This is true for both soundscape and music audio reproduction.

The same cognitive science research described above has also inferred that spatial relationships are more enduring than temporal ones. Aspects of time seem more abstract than those of space because they exist more in the imagination while spatial relationships are easier to perceive as concrete "things" that can be seen or touched. Sound perception is inherently more ineffable because of how sounds are predominantly manifested in the temporal dimension. They come and go and seldom persist for extended durations. This suggests the possibility that musical expression may have served as one means for humans to more directly share and articulate, through organizational intent and manipulation, the experience of the temporal dimension in parallel to the linguistic domain's emphasis on spatial relationships. Are music and audio reproduction, in the absence of words, inverse forms of cross-dimensional asymmetry where temporal representations in some way dominate those of space?

The disorienting experience of hearing temporal resonances — splayed time delayed echoes from extremely separated individual sound sources - confounds normal expectations. It forces us to contend with multiplicities of perception that cannot otherwise simultaneously converge to our attention. There is a confusion of the cues we ordinarily use to perceive time and space. We become aware of many different simultaneous lengths of distance and many different simultaneous events in time. This gestalt is something that neither language nor music can readily do. It also makes it difficult to apply the linguistic domain to further express the unique nature of the experience. The resultant superimposition of multiple space-time frames of reference may be as problematic to the linguistic domain as cross-state retention is 
to a psychedelic experience or our dreams. They all constitute some form of significant juxtaposition and confusion between points of reference in space-time.

\section{Scientific Implications}

Within the past decade, the use of autonomous acoustic sensors for environmental monitoring has grown rapidly among environmental scientists, as a cost-effective, unobtrusive, and scalable solution over on-site human expert observation. Due to constraints on data storage and transmission, contiguous time recordings have been rejected in favor of random or regular sampling methods (recording five or so minutes every hour rather than recording the entire hour). Time averaged acoustic features, such as spectral complexity or spectral density (which collapse many minutes of sound into a single timeaveraged spectrum) are generally used as indicators of ecological factors, such as ecosystem health or biodiversity, rather than their time varying correlates. A single audio frame stands in for the entire extent of many minutes or hours of sonic activity.

When listening to the recordings described in this paper, musicians tend to respond most strongly to the complex system of temporal interactions between sound-makers. Birds and insects call and respond to one another, as well as to non-living and anthropogenic sounds, creating what we perceive as a dense fabric of communication, even when some sounds that appear to interact are too far apart and too quiet to actually be heard by the originating organisms. Considering the wild versus urban environments, the wild recordings tend to invoke a greater sense of mindedness, due to the rich system of interactions between sound makers. In wild environments, anthropogenic sounds, such as automobiles, motorcycles, and airplanes, mask and disrupt this communicative system, which falls into a lull during loud anthropogenic disruptions and then gradually regains momentum afterwards. In the urban environment at UCSC, which had sustained anthropogenic disruption - the continuous sound of the HVAC and overlapping waves of automobile sounds - the communicative system was constantly suppressed. Weeks later, when few to no students were present, the communicative sound networks of non-human living sound-makers could be heard to reassert their presence.

Our experience of listening to the time synchronized multichannel field recordings and related observations about their rich communicative exchange caused us to wonder: is there information in the spatio-temporal structure of the soundscape that might be relevant to ecological concerns and that otherwise time-averaged unsynchronized analysis methods might miss? Is it possible to automatically analyze for and extract features of the temporal structure that capture and quantify the emergent quality of mindedness? What can be gained by considering the soundscape as a dynamical system of interactions 
between sound makers or even as a coupled system of multiple dynamical systems, some nonhuman and others anthropogenic?

Motivated by these questions, we explored some initial attempts to map the soundscape and capture its temporal dynamics, including frequency domain localization, multidimensional cross-similarity matrices, phase-based frequency domain filtering (to look for constructive and destructive interference within certain wavelengths) and unsupervised sound classification.

1. Frequency domain amplitude and phase-based localization: We perform an STFT (short-term Fourier transform) for each microphone array location and compare, across the entire system of locations, the amplitude and phase information for each time-frequency component, giving an estimate of its spatial location. Spatial information is plotted and overlaid on a map of the recording site, producing an animated spatial spectral heatmap that represents how different frequencies of acoustic energy are spread throughout the space. Additionally, we apply frequency domain filters to selectively attenuate or amplify frequencies within selected spatial regions, providing an interactive spatial separation tool. We also apply filters based on phase information to separate frequencies components that exhibit constructive versus destructive interference within certain wavelengths.
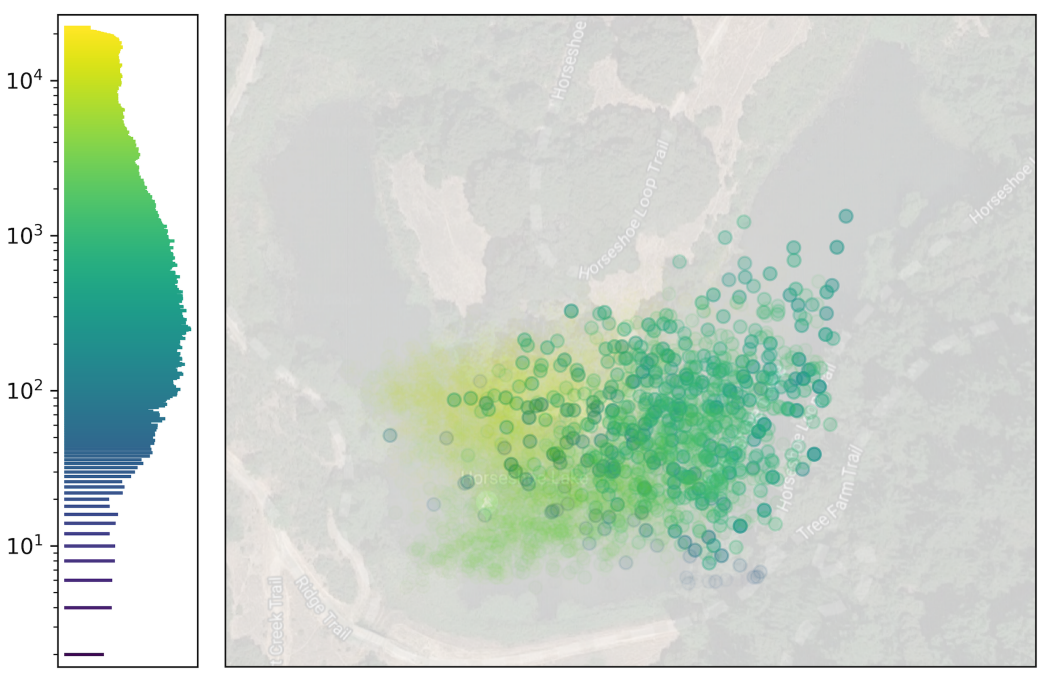

Figure 7: Frequency localization of Horseshoe Lake. Each point represents the estimated spatial location of a single time-frequency component, with frequency indicated by color from low (purple) to high (yellow) and amplitude represented by opacity. 
2. Multidimensional cross-similarity matrices: In order to trace the complex system of temporal relations between similar sounds, we construct a cross-similarity matrix by measuring the spectral similarity between all STFT frames for two different microphone array locations. Doing so for all pairwise combination of locations produces a high-dimensional cross-similarity matrix that represents the time delays when similar sounds occur across microphone array locations. We define a variety of temporal features based on the cross-similarity matrix, which express the makeup and complexity of the temporal structure, including a cross-similarity spectrum, which aggregates the sum total similarity at each time lag of the matrix. Additionally, we construct selfsimilarity matrices for each recording site using a stereo mixdown of all microphones and extract analogous temporal features based on the self-similarity matrix.

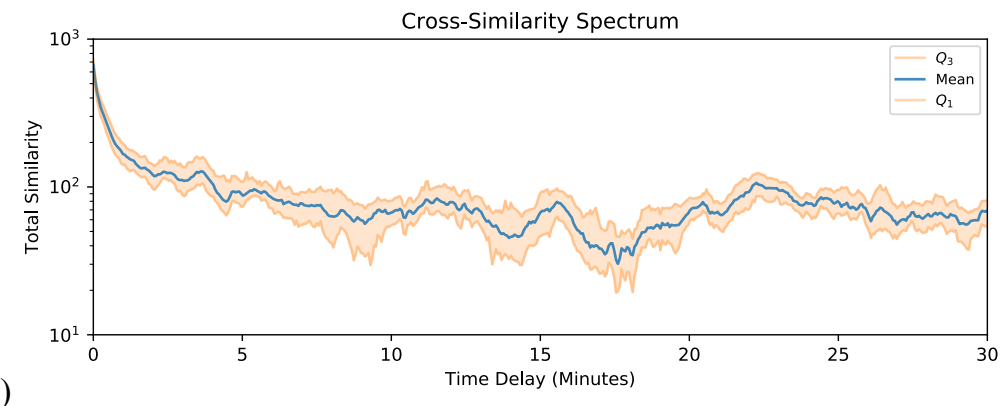

a)

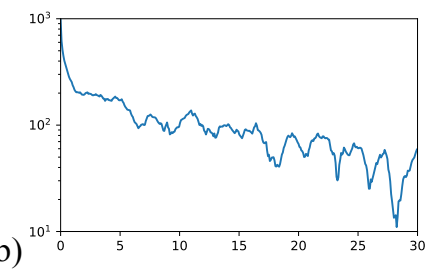

c)
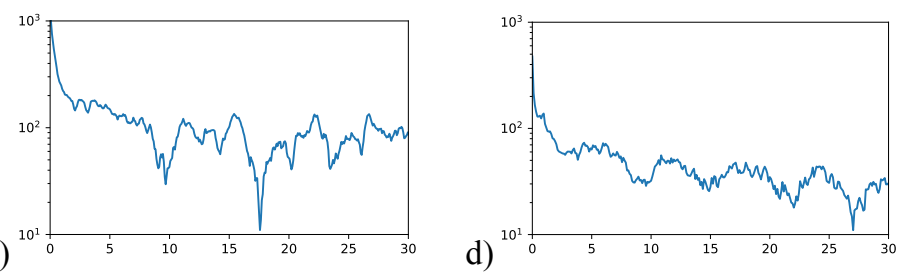

Figure 8: Cross-similarity spectrum of Horseshoe Lake (a) and self-similarity spectra for each of the three recording sites: Alpine Pond (b), Horseshoe Lake (c), and UCSC Campus (d). Horizontal axes represent time delays (in minutes) and the vertical axes represent total similarity at each time delay.

3. Unsupervised embedding: Our goal in this approach is to find a low-dimensional representation of the soundscape's evolution through time and an encoding of its temporal structure. While sound identification is common in bioacoustics - techniques such as spectrogram correlation or matched filters are often employed for the automatic classification of bird song, for instance - the unsupervised classification of soundscape recording is relatively unexplored. We implement an approach based on manifold learning and unsupervised classification to obtain a sequence of discrete states. These states are ultimately represented and analyzed as transition networks. 
First, we encode a frequency-domain representation of a soundscape recording using a lowerdimensional bag-of-words model with a shared vocabulary obtained from a Gaussian Mixture Model (GMM). This representation provides a relatively low-dimensional set of activation vectors, one for each GMM component, that express the soundscape's temporal evolution over time. We embed these sets of activation vectors by windowing into small chunks (on the order of 1 to 5 seconds, with a hop overlap factor of 2 to 8 ) and learning a low-dimensional manifold, or embedding space, using t-distributed Stochastic Neighbor Embedding (t-SNE). To help mitigate the effects of misalignment between chunks, we embed not on the GMM representations directly, but on a transition network representation (low order Markov chain). This embedding represents the gradual (or sometimes abrupt) evolution of temporal patterns over the course of a long ( 1 to 2 hour) recording within a low-dimensional space. The embedding space is segmented into discrete classes of sound behavior using a watershed method, giving a representation of the temporal evolution of the soundscape recording in terms of a sequence of discrete states. Finally, the sequence of discrete states is analyzed and visualized as a transition network graph. Fig. 9 shows transition networks graphs for each microphone location at Horseshoe Lake. Fig. 10 shows a transition network graph for a stereo mixdown of all microphone locations at Horseshoe Lake.

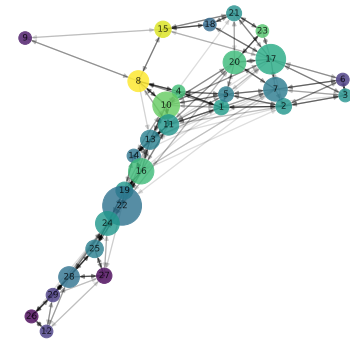

A)

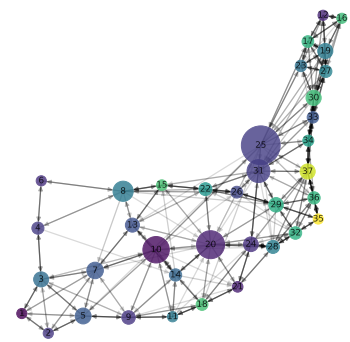

B)

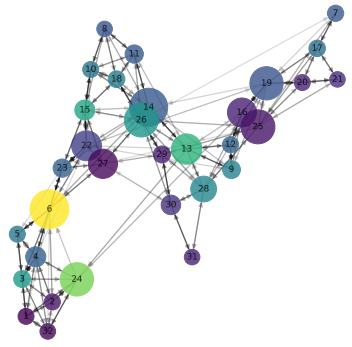

C)

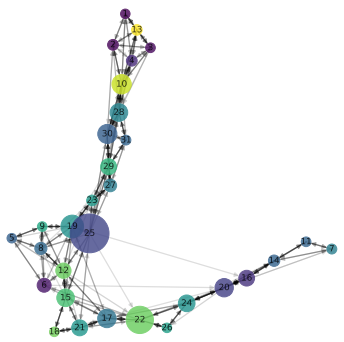

D)

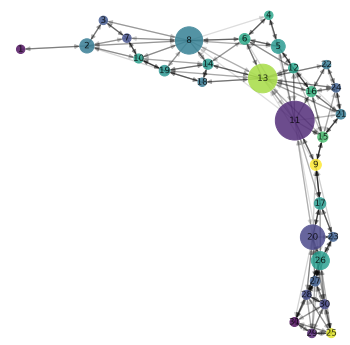

F)

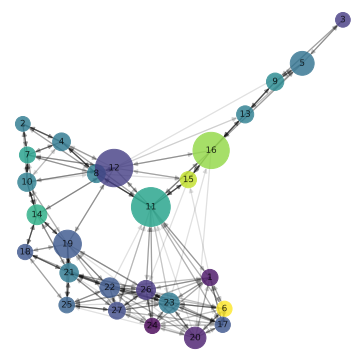

E)

Figure 9: Network analysis of individual Horseshoe Lake recordings. Subfigure letters correspond to microphone locations given in Fig. 4. Node size and color are as in Fig. 10. 


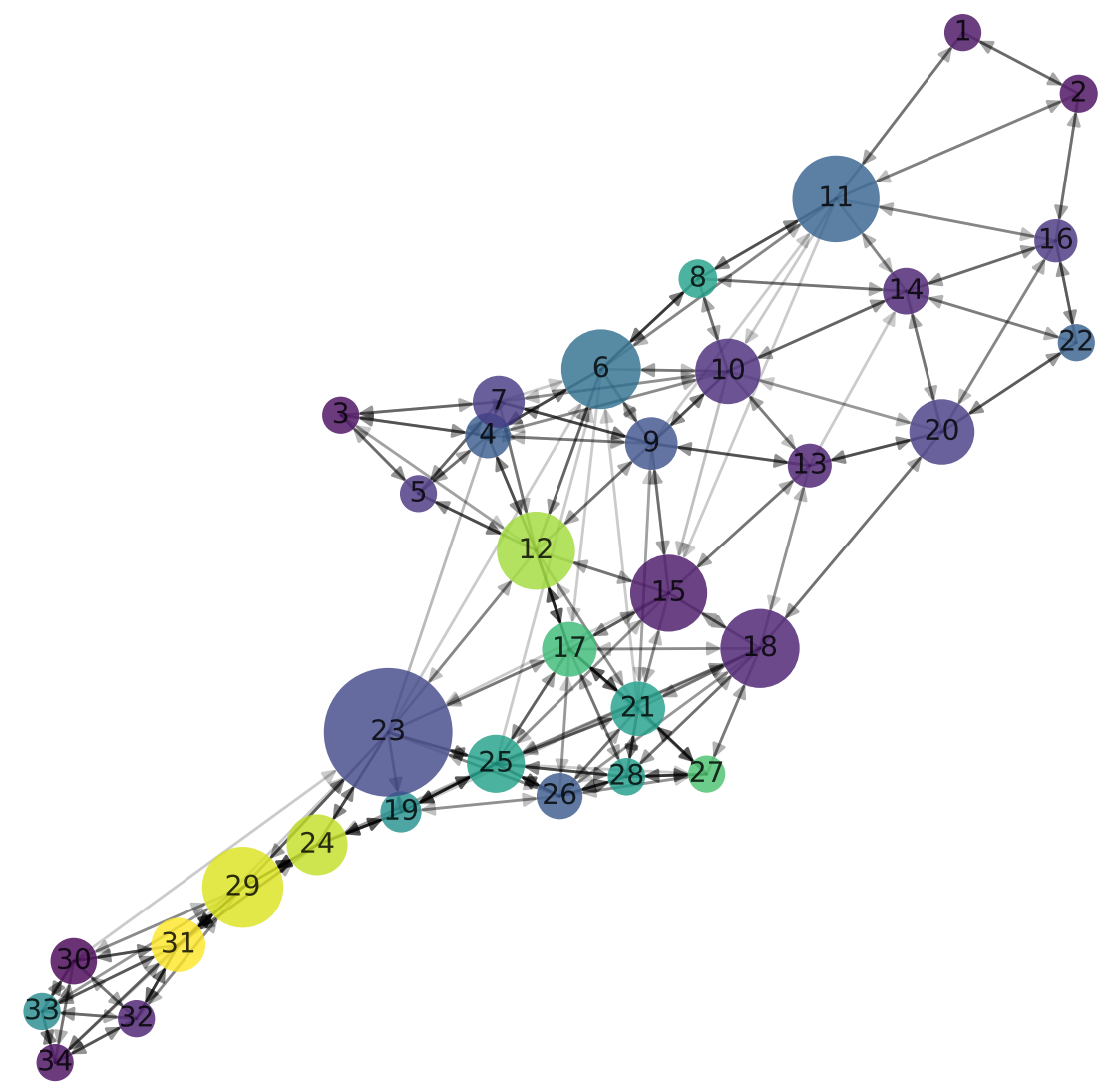

Figure 10: Network analysis of Horseshoe Lake stereo mixdown. Each node (circle) represents a discrete sound behavior class found through embedding and segmentation. Arrows indicate transitions between states. The size of the circle represents the betweenness centrality of each node. The color of each node represents the number of times it is visited, from few (purple) to many (yellow).

\section{Conclusion}

In a traditional audio recording scenario, temporal factors must often be manipulated to facilitate the goal of a final hearing by an ideal listener (POA) and singular frame of reference. In this research scenario, exact time synchronization is maintained while it is space that is manipulated (through compression of distance) to facilitate the representation of a hearing by multiple listeners - as multiple frames of reference - at a large spatial scale. Ideally, this strategy can contribute to the design of new techniques for the collection of bioacoustics data that incorporate a fuller sensory experience of important auditory details. It is also a strategy for bringing auditory anomalies - previously regarded as pejorativeto the foreground in order to acknowledge the profound perceptual challenges they represent. One implication is that this may be a rare situation where our cross-dimensional perception and experience of time and space become more symmetrical and inherently problematic. The novelty, complexity, and —in 
some ways - disorienting reality of the sound worlds represented by this process, appear to challenge our preconditioned habits for listening to audio reproductions and, possibly, aspects of the world at large.

\section{Acknowledgements}

Thanks to the following individuals for contributing in a variety of ways to this research:

Marguerite Brown, Yolande Harris, Melodie Michel, Nathan Ober, Andrew Smith, Andrea Steves, and Dylan Welch.

\section{Photo and Image Credits}

David Dunn (Photos 1, 2; Figures 1-6)

Melodie Michel (Photos 3, 4)

David Kant (Photo 5, Figures 7-10)

\section{References}

Bateson, G., Mind and Nature: A, Necessary Unity, Hampton Press, 2002.

Buonomano, D., Your Brain Is a Time Machine: The Neuroscience and Physics of Time, W.W. Norton, 2017.

Cage, J., Silence: Lectures and Writings, Wesleyan University Press, 1961.

Casasanto, D., Fotakopoulou, O., and Boroditsky, L., Space and Time in the Child's Mind: Evidence for a Cross-Dimensional Asymmetry, Cognitive Science 34, 2010.

Driscoll, J., David Tudor's Rainforest: An Evolving Exploration of Resonance, Leonardo Music Journal, Vol. 14, December, 2004.

Dunn, D., and Crutchfield, J.P., Entomogenic Climate Change, Leonardo Journal, 42:3, 2009.

Dunn, D., Extractions des Espaces Sauvages: Cybernetique de L'ecoute et Ecologie Acoustique. Textes 1981-2008; French translation of writings, Van Dieren Éditeur, Collection Rip on/off, Paris, France, 2011.

Dunn, D., Thresholds and Fragile States, Moebius, Issue 1, University of North Texas, Denton, December, 2012.

Dunn, D., A Philosophical Report From Work-in-Progress (book chapter); Environmental Sound Artists: In Their Own Words, edited by F. Bianchi and V.J. Manzo, Oxford University Press, UK, 2016. 
Eldridge, A., Casey, M., Moscoso, P., and Peck, M., A New Direction for Soundscape Ecology? Toward the Extraction and Evaluation of Ecologically-Meaningful Soundscape Objects using Sparse Coding Methods, PeerJ PrePrints, 2015.

Fristrup, K., and Mennitt, D., Bioacoustical Monitoring in Terrestrial Environments, Acoustics Today, July 2012.

Gasc, A., et al., Assessing biodiversity with sound: Do acoustic diversity indices reflect phylogenetic and functional diversities of bird communities? Ecological Indicators 25, 279-287, February 2013.

Gasc A., et al., Acoustic indices for biodiversity assessments: Analyses of bias based on simulated bird assemblages and recommendations for field surveys, Biological Conservation 191, 306-312, November 2015.

Huber, D.M., Modern Recording Techniques, $9^{\text {th }}$ Edition, Routledge, 2018.

Piaget, J., The Child's Conception of Time, Ballantine Books, 1927/1969.

Piaget, J., The Child's Conception of Movement and Speed, Basic Books, 1946/1970.

Pollan, M., How Does a Writer Put a Drug Trip into Words, NY Times, Dec. 24, 2018.

Snyder, G., and Martin, J., Nobody Home: Writing, Buddhism, and Living in Places, Trinity University Press, 2014.

Turrell, J., Extraordinary Ideas—Realized, Hatje Cantz, August, 2018. 